\title{
Testing and Evaluation of a Augmented Reality System for Battlefield Solders
}

\author{
Jinsuk Kang
}

\begin{abstract}
The purpose of this paper is twofold, first, to provide application direction for NCW (Network Centric Warfare), and second, to suggest development direction for C4I (Command, Control, Communication, Computer) system. In this paper is to develop the context analysis and the interactive platform for intelligent tactical operation in the battlespace. Our research consists of the developments of the HCI and the battlespace awareness scheme based on unstructured data mining, spatial data mining (unstructured data mining) scheme for the smart tactical operation of battalion and below command and control, and the core technology of the battle command system based on the interactive platform.
\end{abstract}

Index Terms-Network centric warfare, battlespace awareness, unstructured data mining, tactical operation.

\section{INTRODUCTION}

Computer technology has come a long way since the days of mainframe systems. Technology has progressed from mainframes and mini-computer to desktop personal computers, to notebooks/laptops, to personal digital assistants (PDA) and handheld computers, to the military computing [1], [2].

Also, as Smartphone are becoming increasingly highly functional and more compact, the core of products is shifting from hardware production technology to software technology. In its first stage, Mobile-phone and PDA just controlled some appliances for industry as a simple control program, but smartphone has evolved to control large and complex appliances such as multimedia processors, battlespace, and military systems. Real-time processing and trustworthy performance are necessary for these systems. In this research presents an online partial 3D modeling methodology that uses a mobile mixed reality system and 3D navigation in the tactical networks(based Internet Protocol), and a tracking methodology that computers the 3D model with a mobile image. Instead of relying on models which are created in advance, the system generates a 3D model for a real building on the navigation by combining frontal and map views. With the battlespace awareness, A user's initial pose is estimated using an landmark (Augmented modeling object), which is retrieved from a database according to the user's GPS coordinates, and an inertial sensor which measures pitch. We detect modeling objects based on graph cut, and find objects and modeling the object by minimizing the proposed cost function. Also, to track the user's position and orientation in real-time, feature-based tracking is carried out based on

Manuscript received August 3, 2016; revised December 1, 2016.

Jin-suk Kang is with Jangwee Research Institute for National Defence, Ajou University, Suwon, Korea (e-mail: jskang01@ajou.ac.kr). salient points on the edges and the feature of a modeling object the user is keeping in view. Battlespace visualization begins with a picture of the ground and successively layers enemy, friendly and environment over terrain visualization [3], [4].

In this paper, the changes in wars of the future want the military to escape from original systems, strategy, tactics, training and logistics. This concept is called a Revolution in Military Affairs (RMA). Recently the military of Korea has been establishing the next generation wireless tactical communication system so called Tactical Information Communication Network (TICN). Hence the purpose of this study is twofold, first, to provide application direction for NCW (Network Centric Warfare), and second, to suggest development direction for C4I (Command, Control, Communication, Computer) system, that coincide in Korean Armed Forces' real condition by analyzing trend of NCW implementation and C4I system construction which advanced nations is implementing on the basis of mixed reality in smartphone concept, new theory of war.

\section{Definitions AND TeChNOLOgY FOR MilitaRy APPLICATIONS}

The impetus for the research arose from studies in which Live, Virtual and Constructive (LVC) techniques were used to supplement live training exercises. A synthetic wrap produced that included virtual aircraft and virtual representations of live ground assets. The virtual aircraft could see and interact with the live participants.

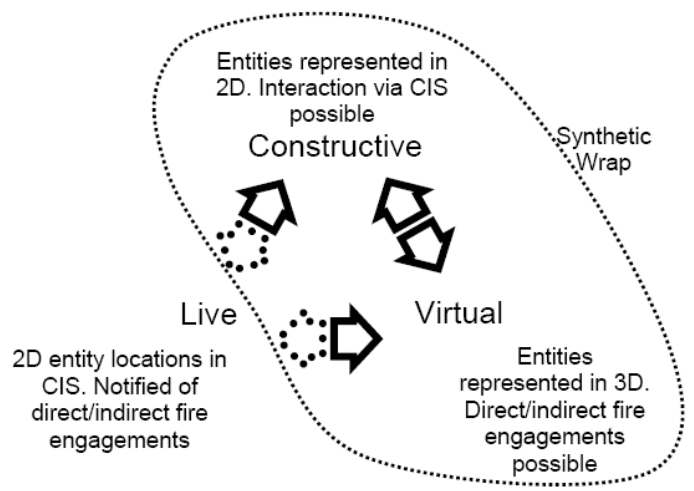

Fig. 1. Connections and capabilities of an application of synthetic wrap.

However, the live participants had no representation of the virtual world. Although geospatial information about the location of the virtual entities could be passed to the live domain via the Command Information System (CIS) and other data links, there was no way of presenting that 
information in the modality that the users were accustomed to i.e. visual, aural, or other platform systems. Fig. 1 gives an overview of the interactions and capabilities of each of the LVC elements. It was realized that a method of stimulating the live domain was needed to support more complex synthetic wrap architectures. This would be fundamental to the provision of vertical training exercises, such as those required for time sensitive target training.

We present a novel approach of collaborative interaction model combining tangible user interface and mobile AR. This model is aware of a user's context through the user-centric integration and inference of contextual information in smart space. Based on the user's context, it filters the content relevant toe the user and overlays the filtered content over the associated physical entities. In addition, the collaboration interaction model generates the community according to the relationship between the entities and enables a user to share the personalized content with other mobile users selectively in a customized way. Also, Development of scale invariant feature transform and general object recognition for tangible user interface service delivery framework in Fig. 2.

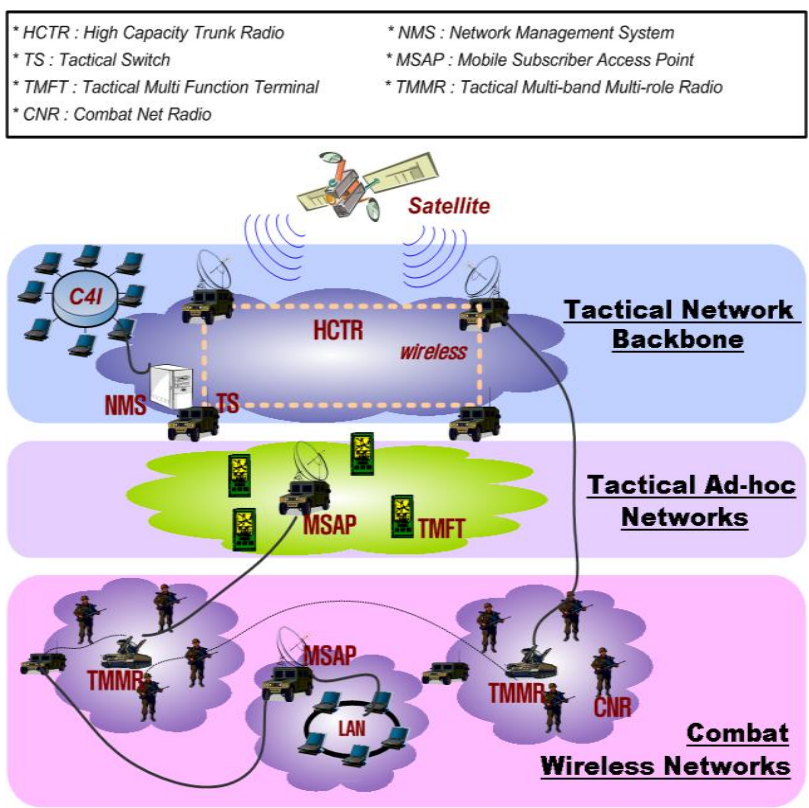

Fig. 2. The sub-structure of tactical communication networks.

\section{SYSTEM OVERVIEW AND SPATIAL INFORMATION, DATABASE}

The objective of this paper is to improve efficiency in 3-dimensional spatial mechanism modeling. For this objective, two points of view are considered broadly. First modeling program has to be user friendly. Second efficient input process has to be provided for easy input of data needed for spatial mechanism. For user-friendly program, graphic user interface can be used. Many commercial dynamic programs use this. In data input process common programs have some disadvantage cam be improved. So in this paper several efficient input processes are suggested and some efficient methods are adapted among many methods used in common program. And for easy modification of complete imputed model efficient modification methods are suggested.

Our AR system relies on computing separate tasks on separate workstations to achieve low latency. One workstation is dedicated to computer vision related tasks, another to simulation and rendering of the synthetic image. Computer generated image is mixed with filmed real image by of the shelf video mixer. There are three main vision related tasks. Calibration of the real camera, tracking of the real camera position, orientation and tracking of objects of interest.

The camera calibration is performed in beforehand and the output is transferred in form of projection matrix to VHD (Virtual Human Director) manually. Camera tracking and object sensing is performed in real-time and the output is transferred via Ethernet to relevant VHD modules. (Object data for simulation and camera parameters for rendering) Our second workstation is running an extended version of VHD for AR. It is performing the virtual environment simulation and rendering. Computer generated image is put on the video output. Fig. 3 illustrates major system components and their relationships.

\section{A. Spatial Information and XML Schemas Database}

The server component has - in addition to the data queried form the Augmented World Model (AWM) - access to a database of general object recognition templates that implement different techniques, as depicted in the bottom of Fig. 3. Each individual general object recognition template available through the database has four functional parts:

1) Part 1: A function to query the template for appropriateness to visualize a specific data type by providing the corresponding data schema.

2) Part 2: Processor to handle raw data objects, provided by the utilized federation.

3) Part 3: The implementation of the actual general object recognition technique.

4) Part 4: I/O routines to transmit or receive all required data of the template's general object recognition.

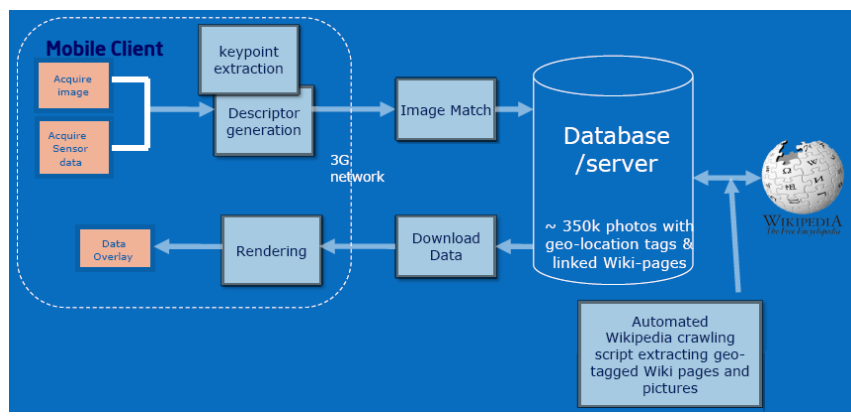

Fig. 3. Complete end-to-end mobile augmented reality system.

In this section, existing road networks stored via GML and the GIS DB composed of information of structures surrounding the roads are used in order to configure a DB for servicing mobile mapping street maps. The required analyses for this mobile service are as seen below. The application schema of the existing DB composed of GML data is as seen in Fig. 4.

Applications using 3D Map DB are generally categorized as Resource Broker sections, which is in charge of geographical information taking into consideration the character of the geographical information that can change at any time, and the Application Layer, which provides real 
screens to the user by using the geographical information. According to the user request, the client program (application layer) requests $3 \mathrm{D}$ geographical information for the area where the user is located to the program that is in charge of the resources (resource broker). The resource broker that received requests for geographical information searches geographical information from the 3D Map DB and the client program converts into file format to provide information that can be understood.

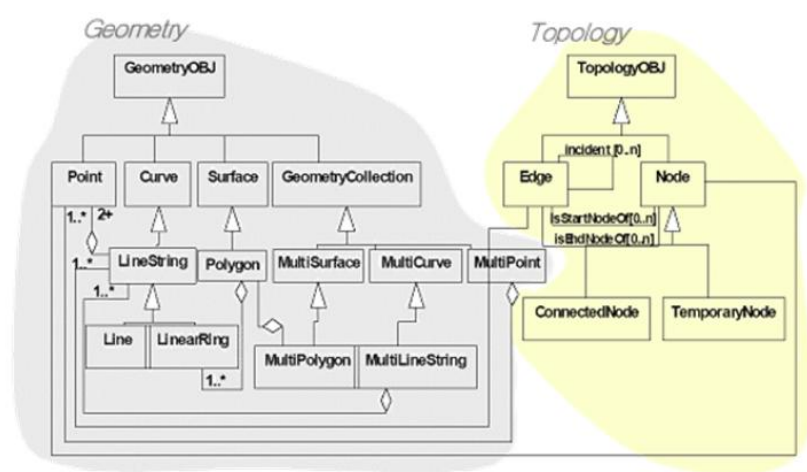

Fig. 4. The proposal of database flowchart.

\section{B. Simulation Model of Unstructured Data Mining Architecture and Tactical Operation}

In this section, presents the unstructured data mining and battle space model, which is capable of propagating various types of emissions from platforms in underwater warfare simulation, predicting interesting encounters between pairs of platforms, and managing environmental information. The unstructured data mining and battle space model has four components: the logger, spatial encounter predictor (SEP), propagator, and geographic information system (GIS) models.

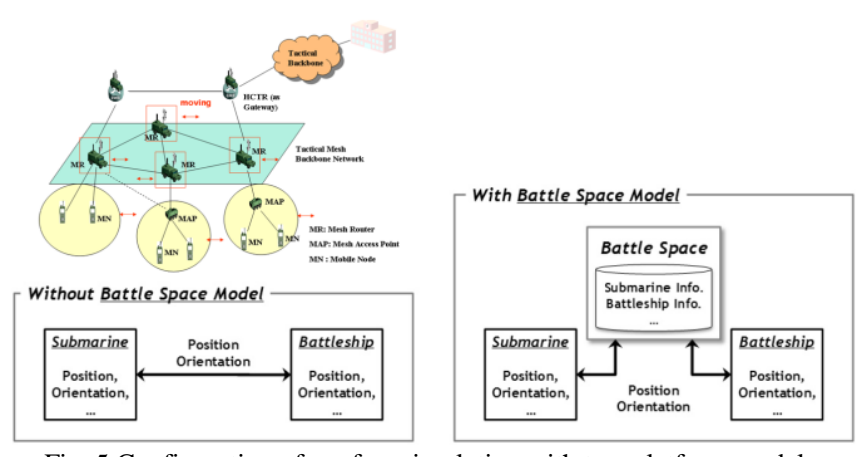

Fig. 5 Configuration of warfare simulation with two platform models.

The logger model stores brief data on all the platforms in the simulation, and the GIS model infers an encounter among the platforms in the simulation, and progresses the simulation to the time when this encounter will happen. The propagator model receives various emissions from platforms and propagates these to other "within-range" platforms by considering the propagation losses and delays. The unstructured data mining and battle space model is based on the discrete event system specification (DEVS) and the discrete time system specification (DTSS) formalisms. To verify the unstructured data mining and battle space model, simple augmented reality warfare between a battlespace and a submarine was simulated. The simulation results with the model were the same as the simulation results without the model Fig. 5.

\section{Simulations And Result Analysis}

The Battle space Modeling and Simulation (BMS) is the Test and Evaluation of a excellence responsibility for Modeling and Simulation (M\&S) responsible for modeling and simulating the behaviors and interactions of elements within the modern battlespace environment. BMS provides credible scenario development, UAS/UAV (Unmanned Aircraft System / Unmanned Aerial Vehicle) expertise and repeatable models of highly complex, interactive and reactive environments that are used across the Department of Defense (DoD) and Naval Aviation Systems Command (NAVAIR) for training, testing, analysis and experimentation.

Our results used a maintains expertise in supporting the Joint Semi-Automated Forces (JSAF), the Simulation System, Joint Integrated Mission Model (JIMM) and the Next Generation Threat System (NGTS). It is actively works with Live, Virtual and Constructive (LVC) Integrated/Distributed Events. The LVC environment provides a common battlefield, on which live units can be represented along with virtual and constructive simulation units. These units interact with one another to conduct a coordinated event (fight, etc.), as though they were physically together on the same ground or in the same air zone. LVC Integrated/Distributed Events utilize protocols (DIS, HLA, TENA), interfaces and virtual battlespace environments (JIMM, NGTS, etc.) to provide realistic, accurate representations of the simulated environment.

\section{A. Battle Space Conceptual Design Model}

Suppose there are three categories of players: a map owner, $N$ firms heterogeneous in quality, and $M$ consumers searching for a desired firm ( $M$ is normalized to 1 without loss of generality). We use a simplified version of Wolinsky [5] in describing the search behavior of consumers, and introducing heterogeneity of firms in a way that broadly follows Chen and $\mathrm{He}$ [6] and Eliaz and Spiegler [7]. A firm's quality (or type) is a number $q$, which is distributed according to a cdf of $G$, with support $[0,1]$. When a consumer is matched with a firm with quality $q$, the match has a positive value for the consumer with probability $q$. And conditional on a positive-value match, the consumer's willingness to pay for the firm's product is a random draw from a distribution of $\operatorname{cdf} F$ and continuous pdf $f$. Consumers search for the first round and might be lured to other firms nearby. This is modeled into a 2-step search process, which is going to be described next. The search costs of consumers and production costs of firms are both abstract from the model. The battle space proceeds as follows. In the first stage, the map owner announces an entry fee $A$ for the potential entering firms and sticks to it thereafter.

Then consumers and firms decide whether to enter the map. Firms that enter pay the entry fee to the map owner and are incorporated into the search pool. In the third stage, firms set price $p$ for their products and consumers start to search in a two-step manner: first they search for a firm that is randomly drawn from the universal firm pool $\{1,2, \ldots, N\}$ for a match; then with some probability, they are lured to the firms in the 
search pool $\{1,2, \ldots, N\}$, where $n$ is the number of firms in the search pool. This search process is illustrated in [Fig. 6]. As illustrated in Fig. 6, after firms set their prices, consumers enter the map and start to search. In the first stage, each consumer randomly draws a firm from universal firm pool. With probability $n / N$ the target firm has entered the map, and in this case, the consumer would be lured by the advertising pool with probability $\alpha$. In the other case that the target firm is not on the map, the consumer's switching probability is $\beta$. Roughly $\alpha$ and $\beta$ measures on average how deterministic consumers are for their first round choice. For consumers who switch to the advertisement pool, they again draw a random firm, but from the advertisement pool instead of the universal firm pool. We multiply a parameter $\gamma$ to the probability of positive value matching in the advertisement pool and assume $\gamma \in[0,1]$. Thus $1-\gamma$ can be interpreted as the general advertisement averse among consumers, which has a negative effect on the probability of successful matches. In other words, $\gamma$ measures on average how much consumers prefer their first round choices to the products in the advertisement pool. And $\{\alpha, \beta, \gamma\}$ constitute the parameters of our interest.

Real life scenarios motivate the search process described above. A key difference with the tradition search engine is that, in a digital map the search pool and the advertising pool are by nature. For a firm that is labeled on the map, it shows up automatically when consumers search nearby firms. We stress this difference by comparing digital maps with traditional search engines in Fig. 7. In this paper we focus on the case of a monopolistic map owner, but the case of duopoly or more general cases could also be analyzed within this framework.

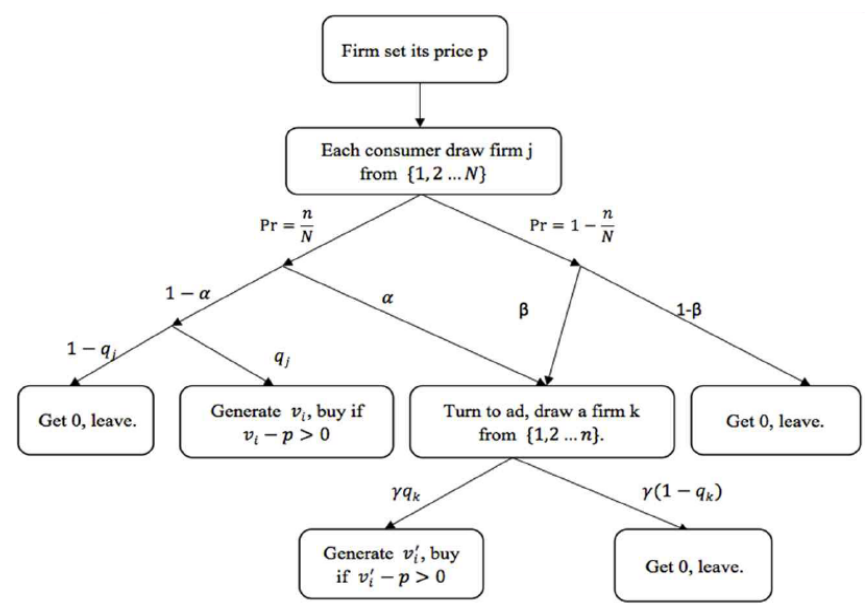

Fig. 6. The searching process.

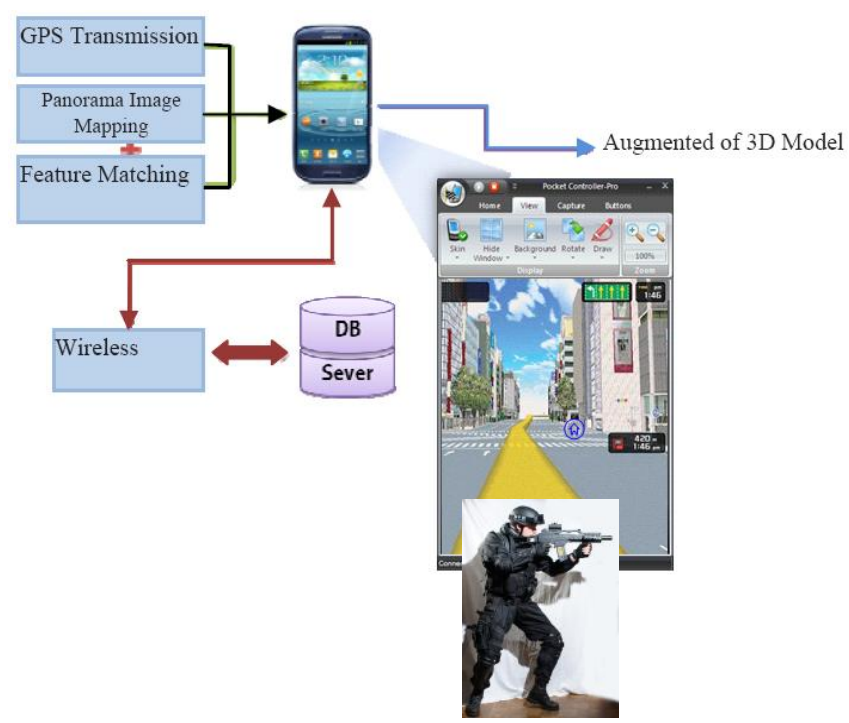

Fig. 7. Experiment using the mobile device of a target object within screen shots cadastral information service.

\section{B. References}

Proposed 3D model visualization system of battle space sites is as shown on Fig. 8. Fig. 8 shows 3D model visualization process of battle space sites through feature point matching of image of battle space sites inputted from smart phone camera and stored object panorama image. Android 2.3.4 developed by Google through use of minimum system implementation environment was used. Library used for battle space sites 3D model visualization system is as shown on below Table I.

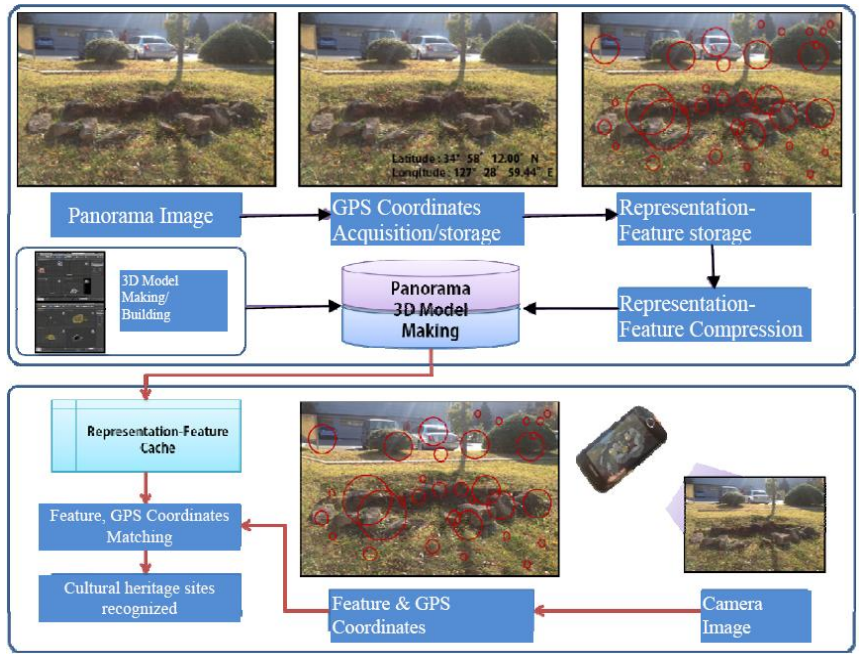

Fig. 8. Space sites 3D visualization system process

For 3D library, Parser library that may load 3D object, object movement and core library that enables texture mapping were used together with camera review and rendering of 3D object. Through above visualization system process as shown on Fig. 8, 3D model visualization system of 
battle space sites based on outdoor augmented reality was implemented. Also, 3D model was augmented in battle space sites by using smart phone camera. In diversified angles of camera, free augmentation of 3D model is allowed.

TABLE I: INITIAL VALUES OF AUGMENTED REALITY WARFARE SIMULATION

\begin{tabular}{c|c}
\hline Variables & Values \\
\hline Torpedo & $25 \mathrm{~km}$ \\
\hline Search pattern & Snake search \\
\hline Detection range & $1,500 \mathrm{~m}$ \\
\hline Ping repetition & $2 \mathrm{~s}$ \\
\hline Beam width (transmission) & $30^{\circ}$ \\
\hline Beam width (reception) & $40^{\circ}$ \\
\hline Target speed & $15 \mathrm{~km}$ \\
\hline Target dimension & $80 \times 7 \times 7 \mathrm{~m}$ \\
\hline (Length x Breadth x Depth) & $3,000 \mathrm{~m}$ \\
\hline Maximum attach range & $6 \% \mathrm{sec}$ \\
\hline Turn rate & $30^{\circ}$ \\
\hline Sweep angle & $0.05 \mathrm{sec}$ \\
\hline Unit simulation time &
\end{tabular}

\section{CONCLUSION}

We have proposed a model to describe the behavior of advertisement on tactical operation for battle space. In the modern warfare, the importance of battlefield combat is now growing bigger than ever before. In this paper, we propose the efficient methods for utilizing the combat training warfare by focusing on the tactical operation. The real-time GIS engine can provide insert, delete, update, search operations of spatial data, in addition to operations of the existing real-time database system. And, it can deal with spatial and aspatial queries and manage spatial and aspatial indices for the efficient management of information of user's locations. The real-time GIS engine consisted of a data loader which can load data from back-end GIS to the real-time GIS engine, a location data manager which can distribute location data over several systems, a layer manager which can manage layers, and so on.

This system uses automatic segmentation based on coordinates and geographic features as well as an indexing structure, which is a variation of the frame-segment tree. It generates video-maps i.e. video with superimposed information of geographic features, creating a map where a video is on the bottom layer. It is also able to generate video-itineraries in response to spatial queries, i.e. a composition of sequences showing the geographic features that satisfy the query. We propose video-maps and video-itineraries as an alternative interface to traditional maps for non-skilled users.

\section{ACKNOWLEDGMENT}

This research was supported by Basic Science Research Program through the National Research Foundation of Korea (NRF) funded by Ministry of Education No. 2014R1A2A1A11049469.

\section{REFERENCES}

[1] Military Technology: MILTECH, EUROSATORY FOCUS, vol. XXXII, issue 6, Jan. 2008.

[2] J. S. Kang, C. H. Park, J. H. Kim, and Y. S. Choi, "Implementation of embedded system vehicle tracking and license plate recognition using spatial relative distance," in Proc. $26^{\text {th }}$ International Conference on Information Technology Interface, June, 2004, pp. 167-172.

[3] J. S. Kang, T. T. Jeong, S. H. Oh, and M. Y. Sung, "Image streaming and recognition for vehicle location tracking using mobile devices," Springer LNCS 4459, pp. 730-737, 2007.

[4] J. S. Kang, Y. H. You, and M. Y. Sung, "Design of MMS(Mobile Mapping Systems) applicable to LBS(Local Based Services)," in Proc of IEEE ICDIM, Oct. 2007, pp. 487-495.

[5] A. Wolinsky, "True monopolistic competition as a result of imperfect information," Quarterly Journal of Economics, vol. 101, no. 3, pp. 493-511, 1986.

[6] K. Eliaz and R. Spiegler, "A simple model of search engine pricing," The Economic Journal, vol. 121, no. 556, pp. 329-339, 2011.

[7] C. J. Combrink and J. J. Nel, "The application of modeling and simulation to expand the user body of knowledge as part of acquisition support," INCOSE South Africa, Pretoria, August 2006.

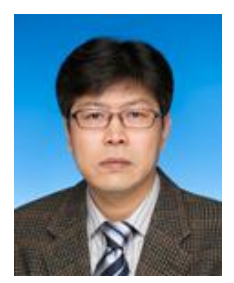

Jinsuk Kang received his B.S. degree in information engineering from Cheju National University, Jeju, Korea, in 1999 and his M.S. and Ph.D. degrees in computer engineering from Cheju National University, Jeju, Korea, in 2001 and 2005, respectively. From 2006 to 2009, he was with the university of incheon, Korea, as a research professor. From February 2009 to March 2010, he worked with Chungbuk National University, Korea, as a visiting professor. Since March 2010, he has been with the Jangwee Research Institute for National Defence, Ajou University, Suwon, Korea, where he is currently a Research professor. His research interests include the areas of multimedia, computer vision, human-computer interaction, mobile computing and embedded system, etc. 